\title{
Density of partition function zeroes and phase transition strength
}

\author{
Wolfhard Janke ${ }^{\text {a }}$, Ralph Kenna ${ }^{\text {b,* }}$ \\ a Institut für Theoretische Physik, Universität Leipzig, Augustusplatz 10/11, 04109 Leipzig, Germany \\ ${ }^{\mathrm{b}}$ School of Mathematics, Trinity College Dublin, Ireland
}

\begin{abstract}
A new method to extract the density of partition function zeroes (a continuous function) from their distribution for finite lattices (a discrete data set) is presented. This allows direct determination of the order and strength of phase transitions numerically. Furthermore, it enables efficient distinguishing between first and second order transitions, elucidates crossover between them and illuminates the origins of finite-size scaling. The efficacy of the technique is demonstrated by its application to a number of models in the case of Fisher zeroes and to the $X Y$ model in the case of Lee-Yang zeroes. (C) 2002 Elsevier Science B.V. All rights reserved.
\end{abstract}

PACS: 64.60.-i; 68.35.Rh; 05.70.Fh; 05.50.+q

Keywords: Density of partition function zeroes; Phase transitions; Finite-size scaling; Latent heat; Critical exponents

\section{Introduction}

Phase transitions are phenomena common to a wide range of disciplines, from physics to biology, economics and even sociology. Examples include neural networks, protein folding, melting, magnetism, stock market crashes and the deconfinement transition in the early universe.

In statistical physics, in particular, one is interested in the determination of the location, order and strength of phase transitions. First order transitions involve the coexistence of two or more distinct phases and are characterized by a discontinuity in the first derivative of the free energy corresponding to the finite amount of energy needed to transform from one phase to the other. For temperature driven transitions, this

\footnotetext{
* Corresponding author.

E-mail address:rak@maths.tcd.ie (R. Kenna).
}

discontinuity is the latent heat $\Delta e$ and is a measure of the transition strength. For second order transitions there is no such coexistence. Instead, thermodynamic quantities such as the correlation length and specific heat diverge. Such divergences are characterized by critical exponents $v$ and $\alpha$, which is a measure of the strength of the transition.

The computational approach to the study of phase transitions consists of two steps-the gathering of data in the form of a Monte Carlo simulation followed by a numerical analysis of appropriate quantities. The first of these is restricted to systems of finite size. Phase transitions, however, require an infinite number of available states for their occurrence. The second step in the numerical approach is an extrapolation to infinite volume. Traditional techniques involve the finitesize scaling (FSS) study of thermodynamic functions. An increasingly popular alternative approach is, however, the use of zeroes of the partition function. 
Let $t=T / T_{c}-1$ be the reduced temperature and $h$ the external field. For a $d$-dimensional system of linear extent $L$, the FSS of the $j$ th complex partition function zero (for large $j$ ) is given by [1]

$t_{j}(L) \sim\left(j / L^{d}\right)^{1 / v d}$,

or

$h_{j}(L) \sim\left(j / L^{d}\right)^{(d+2-\eta) / 2 d}$.

Here $\eta$ is the anomalous dimension and $h=0$ in the first formula (where the zeroes are called Fisher zeros) while $t=0$ in the second (where the zeroes are LeeYang zeroes). The standard approach to FSS of zeroes is to fix the index to $j=1$ and extract an estimate for the critical exponents from a range of lattice sizes. It has, however, long been known that using more than one index could provide more information. However, since (1) and (2) are inexact, this has been prohibitive. In particular, the extraction of the density of zeroes (a continuous function) from their (discrete) distribution for a finite and numerically accessible lattice has been considered prohibitively difficult or even impossible [2]. In recent years, however, there have been some attempts to overcome the difficulties involved [3]. In view of the increasing importance attached to this approach, we suggest an appropriate way this should be done [4].

\section{Density of zeroes}

The partition function for finite $L$ is $Z_{L}(z) \propto$ $\prod_{j}\left(z-z_{j}(L)\right)$, where $z$ is an appropriate function of temperature or field. We assume the zeroes, $z_{j}$, are on a line impacting on to the real axis at the critical point, $z_{c}$. Parameterizing zeroes on this line by $z_{j}=z_{c}+r_{j} \exp (\mathrm{i} \varphi)$ we may define the density of zeroes as $g_{L}(r)=L^{-d} \sum_{j} \delta\left(r-r_{j}(L)\right)$. The cumulative distribution function of zeroes is then $G_{L}(r)=\int_{0}^{r} g_{L}(s) \mathrm{d} s$ which is $j / L^{d}$ if $r \in\left(r_{j}, r_{j+1}\right)$. At a zero we assume the cumulative density is given by the average $[5,6]$

$G_{L}\left(r_{j}\right)=(2 j-1) / 2 L^{d}$.

In the thermodynamic limit and for a phase transition of first order this integrated density of zeroes is, in fact [5],

$G_{\infty}(r)=g_{\infty}(0) r$, so that the density is non-vanishing at the real axis. The slope at the origin in (4) is related to the latent heat in the Fisher case (or magnetization in the LeeYang case) via [5]

$g_{\infty}(0) \propto \Delta e$.

For a second order transition the corresponding expressions for Fisher and Lee-Yang zeroes are [7]

$G_{\infty}(r) \propto r^{2-\alpha} \quad$ or $\quad G_{\infty}(r) \propto r^{2 d /(d+2-\eta)}$,

respectively.

Thus while the scaling behaviour of the position of the first few zeroes in the complex temperature plane can be used to identify $v$ via (1), the density of zeroes gives the strength of the transition. A plot of $G_{L}\left(r_{j}\right)$ against $r_{j}(L)$ should (i) go through the origin, (ii) display $L$ - and $j$-collapse and (iii) reveal the order and strength of the phase transition by its slope near the origin.

In (3), $r_{j}$ may be taken to be the imaginary part of the position of the $j$ th zero. Equating (3) to (6) in the second order Fisher case, gives $r_{j}(L) \sim L^{-1 / \nu}$. This is the usual FSS formula for fixed index Fisher zeroes. Similarly, in the Lee-Yang case, one recovers the fixed index FSS formula $h_{j}(L) \sim L^{-(d+2-\eta) / 2}$. Also, equating (3) to (4) gives $r_{j}(L) \sim L^{-d}$, explaining the usual identification of $v$ with $1 / d$ for a first order temperature driven phase transition. Therefore, traditional FSS emerges quite naturally from this density approach.

\section{Applications}

To demonstrate our approach, we perform fits to the cumulative density of zeroes for a number of different models in statistical physics and in lattice field theory [4]. Here we present the results for the Fisher zeroes for two models, one from each field. Furthermore, we discuss the Lee-Yang zeroes of the two-dimensional $X Y$ model.

Allowing for first or second order behaviour, the cumulative density should behave as

$G(r)=a_{1} r^{a_{2}}+a_{3}$,

where we also allow for an additional parameter $a_{3}$ which should be zero for a good fit. In fact, a non-zero value of $a_{3}$ indicates the absence of a phase transition, 


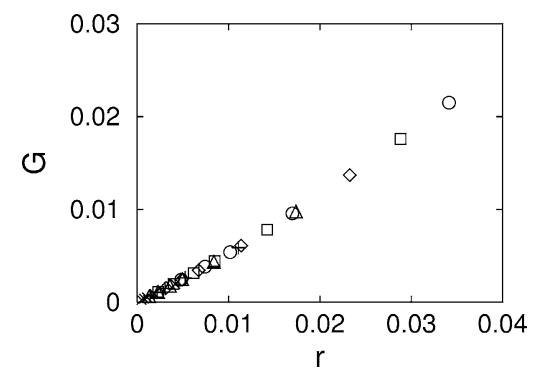

Fig. 1. Distribution of zeroes for the $d=2, q=10$ Potts model (for $L=16-64$ ) which has a first order phase transition. The symbols $\times,+, \triangle, \diamond, \square$, and $\circ$, correspond to $j=1,2,3,4,5$, and 6 , respectively.

for, $a_{3}>0$ means the zeroes have already crossed the real axis (the situation in the broken phase) while $a_{3}<0$ means the zeroes have not yet reached the real axis (the symmetric phase). For Fisher zeroes, a first order phase transition is indicated if $a_{2} \sim 1$ for small $r$, in which case the latent heat is proportional to the slope $a_{1}$. A value of $a_{2}$ larger than 1 signals a second order transition whose strength is given by $\alpha=2-a_{2}$. Note that $\alpha$ can be measured directly using this method while traditional FSS only allows the measurement of the ratio $\alpha / \nu$.

The $d=2, q=10$ Potts model: The first six Fisher zeroes for the two-dimensional 10-state Potts model for lattice sizes $L=4-64$ are listed in [8]. A traditional FSS analysis applied to the first zero for large lattices provides evidence for $v=1 / d$ and hence a first order phase transition. However, the determination of the lattice size above which FSS sets in is, by necessity, somewhat arbitrary. Indeed, when one extends the analysis to higher index zeroes one finds that when corrections are ignored, no two-parameter fit gives an acceptable result.

Our analysis of the density of zeroes begins with Fig. 1. The excellent data collapse for various $L$ and $j$ indicates that (3) is the correct form for the density of zeroes. Fitting (7) to the $L=16-64, j=1-4$ data points gives $a_{2}=1.10(1)$ and $a_{3}=0.00004(1)$, indicating a first order phase transition. Fixing $a_{3}=0$, $a_{2}=1$ and applying a single-parameter fit close to the origin yields a slope corresponding to latent heat $\Delta e=0.698$ (2) which compares well with the exact value 0.6961 .

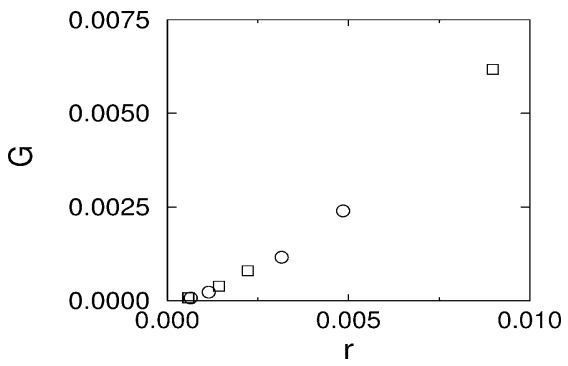

Fig. 2. Distribution of zeroes for the four-dimensional Abelian surface gauge model which has a second order transition. The symbols $\square$ and $\circ$ correspond to the $j=1$ and $j=2$ index zeroes, respectively.

The $d=4$, Abelian surface gauge model: This is a model dual to the $d=4$ Ising model, which, up to logarithmic corrections has mean field critical exponents [9]. One therefore expects the surface gauge model also to be characterized by mean field exponents with $\alpha=0$ and $v=1 / 2$.

The first two Fisher zeroes for lattices of size $L=3$ to 12 are listed in [10] where a conventional analysis applied to the first index zero yields the best estimate of $v=0.469(17)$ from the two largest lattices. Inclusion of the smaller lattices worsens the fit driving $v$ away from $1 / 2$. Also, a bimodal structure in the energy histograms appears as a spurious indication of a first order transition [10].

A fit of the data to (7) yields $a_{2}$ incompatible with unity (see Fig. 2), with a fit near the origin yielding $a_{2}=1.90$ (9). This corresponds to $\alpha=0.10(9)$, compatible with zero. Note that only the region near the origin in Fig. 2 is of interest. The slope there is not compatible with a first order transition and the bimodal structure of the energy histograms observed in [10] can only be an unexplained finite-size effect.

The $d=2 X Y$ model: $\quad$ Here we demonstrate that the density technique is also applicable in the Lee-Yang case. The first few Lee-Yang zeroes for the $d=2$ $X Y$ model with $L=32-256$ were determined for various temperatures in Ref. [11]. Fig. 3 depicts the distribution of these zeroes for $\beta=1 / k_{\mathrm{B}} T=1.113$ which is the critical point (here $k_{\mathrm{B}}$ is the Boltzmann constant). From (6), and with $\eta=1 / 4$ (the value of the anomalous dimension in this model), one expects $G_{L}\left(r_{j}\right) \sim r_{j}^{16 / 15}$, where $r_{j}=h_{j}$. A three-parameter fit to (7) gives $a_{3}=0$ indicating that criticality has indeed 


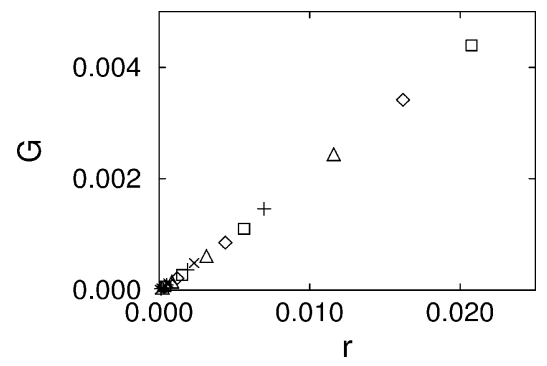

Fig. 3. Distribution of Lee-Yang zeroes for the two-dimensional $X Y$ model at $\beta=1.113$ (for $L=32-256$ ). The symbols $\times,+, \triangle, \diamond$, and $\square$ correspond to $j=1,2,3,4$, and 5 , respectively.

been reached at this temperature. A two-parameter fit now yields $a_{2}=1.06(1)$, compatible with expectation. One notes that logarithmic corrections are present in this model as shown in $[11,12]$.

\section{Conclusions}

We have presented a new method to extract the (continuous) density of zeroes from (discrete) finitesize data and demonstrated how this can be used to distinguish between phase transitions of first and second order as well as to measure their strengths. The method meets with a high degree of success in statistical physics and lattice field theory and lends new insights into the origins of finite-size scaling.

\section{References}

[1] C. Itzykson, R.B. Pearson, J.B. Zuber, Nucl. Phys. B 220 (1983) 415
[2] P.P. Martin, Nucl. Phys. B 220 (1983) 366; Nucl. Phys. B 225 (1983) 497.

[3] R. Kenna, C.B. Lang, Phys. Rev. E 49 (1994) 5012;

H. Simon, M. Baake, U. Grimm, in: C. Janot, R. Mosseri (Eds.), Proceedings of the 5th International Conference on Quasicrystals, World Scientific, Singapore, 1995, p. 100; Ch. Binek, Phys. Rev. Lett. 81 (1998) 5644;

R.J. Creswick, S.-Y. Kim, in: D.P. Landau, K.K. Mon, H.-B. Schüttler (Eds.), Computer Simulation Studies in Condensed Matter Physics X, Springer, Berlin, 1998, p. 224; Comp. Phys. Comm. 121 (1999) 26;

R. Burioni, D. Cassi, L. Donetti, J. Phys. A 32 (1999) 5017.

[4] W. Janke, R. Kenna, J. Stat. Phys. 102 (2001) 1211.

[5] C.N. Yang, T.D. Lee, Phys. Rev. 87 (1952) 404; Phys. Rev. 87 (1952) 410

[6] P. Butera, M. Comi, G. Marchesini, E. Onofri, Nucl. Phys. B 326 (1989) 758;

P.H. Damgaard, U.M. Heller, Nucl. Phys. B 410 (1993) 494;

K.-C. Lee, Phys. Rev. Lett. 73 (1994) 2801;

V. Matveev, R. Shrock, Phys. Lett. A 204 (1995) 353;

M. Gürtler, E.-M. Ilgenfritz, A. Schiller, Phys. Rev. D 56 (1997) 3888.

[7] R. Abe, Prog. Theor. Phys. 38 (1967) 322;

M. Suzuki, Prog. Theor. Phys. 38 (1967) 1243.

[8] R. Villanova, Ph.D. Thesis, Florida State University, 1991 (unpublished);

R. Villanova, N.A. Alves, B.A. Berg, Nucl. Phys. B (Proc. Suppl.) 20 (1991) 665; Phys. Rev. B 43 (1991) 5846.

[9] R. Kenna, C.B. Lang, Phys. Lett. B 264 (1991) 396; Nucl. Phys. B 393 (1993) 461; Erratum, Phys. Lett. B 411 (1994) 340.

[10] M. Baig, R. Villanova, Phys. Lett. B 333 (1994) 445.

[11] R. Kenna, A.C. Irving, Phys. Lett. B 351 (1995) 273; Phys. Rev. B 53 (1996) 11 568; Nucl. Phys. B 485 (1997) 583.

[12] A. Patrascioiu, E. Seiler, Phys. Rev. B 54 (1996) 7177; M. Campostrini, A. Pelissetto, P. Rossi, E. Vicari, Phys. Rev. B 54 (1996) 7301;

W. Janke, Phys. Rev. B 55 (1997) 3580. 